\title{
Construction of a plant-transformation-competent BIBAC library and genome sequence analysis of polyploid Upland cotton (Gossypium hirsutum L.)
}

Mi-Kyung Lee ${ }^{1 \dagger}$, Yang Zhang ${ }^{1 \dagger}$, Meiping Zhang ${ }^{1,2 \dagger}$, Mark Goebel ${ }^{1}$, Hee Jin Kim³ ${ }^{3}$ Barbara A Triplett ${ }^{3}$, David M Stelly ${ }^{1}$ and Hong-Bin Zhang ${ }^{1 *}$

\begin{abstract}
Background: Cotton, one of the world's leading crops, is important to the world's textile and energy industries, and is a model species for studies of plant polyploidization, cellulose biosynthesis and cell wall biogenesis. Here, we report the construction of a plant-transformation-competent binary bacterial artificial chromosome (BIBAC) library and comparative genome sequence analysis of polyploid Upland cotton (Gossypium hirsutum L.) with one of its diploid putative progenitor species, G. raimondii Ulbr.

Results: We constructed the cotton BIBAC library in a vector competent for high-molecular-weight DNA transformation in different plant species through either Agrobacterium or particle bombardment. The library contains 76,800 clones with an average insert size of $135 \mathrm{~kb}$, providing an approximate $99 \%$ probability of obtaining at least one positive clone from the library using a single-copy probe. The quality and utility of the library were verified by identifying BIBACs containing genes important for fiber development, fiber cellulose biosynthesis, seed fatty acid metabolism, cotton-nematode interaction, and bacterial blight resistance. In order to gain an insight into the Upland cotton genome and its relationship with G. raimondii, we sequenced nearly 10,000 BIBAC ends (BESs) randomly selected from the library, generating approximately one BES for every $250 \mathrm{~kb}$ along the Upland cotton genome. The retroelement Gypsy/DIRS1 family predominates in the Upland cotton genome, accounting for over $77 \%$ of all transposable elements. From the BESs, we identified 1,269 simple sequence repeats (SSRs), of which 1,006 were new, thus providing additional markers for cotton genome research. Surprisingly, comparative sequence analysis showed that Upland cotton is much more diverged from G. raimondii at the genomic sequence level than expected. There seems to be no significant difference between the relationships of the Upland cotton D- and Asubgenomes with the $G$. raimondii genome, even though $G$. raimondii contains a D genome $\left(D_{5}\right)$.

Conclusions: The library represents the first BIBAC library in cotton and related species, thus providing tools useful for integrative physical mapping, large-scale genome sequencing and large-scale functional analysis of the Upland cotton genome. Comparative sequence analysis provides insights into the Upland cotton genome, and a possible mechanism underlying the divergence and evolution of polyploid Upland cotton from its diploid putative progenitor species, G. raimondii.
\end{abstract}

Keywords: BIBAC library, Gossypium hirsutum, Gossypium raimondii, BIBAC end sequence (BES), Genome evolution, SSR, Polyploidization and evolution

\footnotetext{
* Correspondence: hbz7049@tamu.edu

${ }^{\dagger}$ Equal contributors

'Department of Soil and Crop Sciences, 2474 TAMU, Texas A\&M University,

College Station, TX 77843-2474, USA

Full list of author information is available at the end of the article
} 


\section{Background}

Cottons (Gossypium L. species) are the leading fiber and an important oilseed crop in the world. Cotton fibers sustain the world's textile industry and are an alternative of the synthetic fibers whose production annually consumes billions of barrels of fossil oil worldwide. Cottonseeds are traditionally used to produce food oil and currently have been used as the feedstock of biodiesel production. Furthermore, cottons are also a model system for studies of plant polyploidization, cellulose biosynthesis and cell wall biogenesis. The cultivated cottons, Upland cotton (G. hirsutum L.) and Sea Island cotton (G. barbadanse L.), are allotetraploids containing two homoeologous genomes defined A- and D-subgenomes. It was estimated that they originated from their diploid progenitor species about $1-2$ million years ago [1-3]. Therefore, the cotton polyploiddiploid complex has been widely used as a model system for study of plant polyploidization and its impacts on speciation, biology and evolution $[4,5]$. Cotton fibers, usually 20-40 mm long and $15 \mu \mathrm{m}$ thick, are derived from individual epidermal cells of developing seeds and more than $90 \%$ of their content is cellulose. Cellulose is a major component of plant cell walls and constitutes the largest portion of plant biomass, with an estimated annual world production of billions of metric tons. Therefore, cotton research is of significance not only for the world's textile and energy industries, but also for understanding the mechanism underlying cellulose biosynthesis and cell wall biogenesis [6-9] that are applicable to the entire plant kingdom.

Cottons have been subjected to extensive research in modern genomics and genetics [10]. A number of molecular genetic maps and several thousands of DNA markers have been developed, hundreds of genes and QTLs (quantitative trait loci) of agronomic importance have been mapped, and a large collection of expressed sequence tags (ESTs) have been generated from a variety of tissues collected at different growth and development stages [10]. Bacterial artificial chromosome (BAC) libraries have been demonstrated to be crucial for different aspects of advanced genomics and genetics research, and have been constructed for some genotypes of Upland cotton [11-14] and Sea Island cotton [15]. A draft physical map [16] and draft genome sequences (http://www.ncbi.nlm.nih.gov/ sra/SRA024364? report $=$ full) have been generated recently for G. raimondii. Gossypium raimondii contains a D genome $\left(D_{5}\right)$ and was proposed to be the closest diploid progenitor of the D-subgenome of allotetraploid cottons, including Upland cotton [4]. Nevertheless, no large-insert plant-transformation-competent binary BAC (BIBAC) library has been reported yet for any of the cotton species. BIBAC libraries have all of the functionality of BAC libraries, but also can be used as a vehicle of large DNA fragments containing a number of genes and QTLs for direct transformation in plants via either Agrobacterium [17-21] or biolistic bombardment [22] because they have their own selection markers for plant transformation. The direct transformation competence of BIBACs streamlines their uses for gene and QTL cloning [23], large-scale genome functional analysis [19,22,24] and molecular breeding. For instance, using BIBACs spanning the interval of a QTL (e.g., 5 - $10 \mathrm{cM}$ ), the DNA fragment containing the QTL could be identified by BIBAC transformation, without the need of high-resolution mapping used in the traditional but laborious process of map-based cloning. Using BIBACs with an average insert size of about $150 \mathrm{~kb}$ as a vehicle, the Upland cotton genome could be directly transformed into another genome by approximately 16,000 transformations, which is within the realm of current transformation technologies $[17,19,22]$. These utilities of BIBACs will dramatically facilitate many aspects of current genomics and genetics research such as gene and QTL cloning, targeted marker development and molecular breeding. Moreover, transformation of BIBACs with an insert size of about $150 \mathrm{~kb}$ potentially containing 5-30 genes allows transferring in a single event a cluster of genes that are likely involved in a particular biological process, thus improving the efficiency of molecular breeding [22,24]. Finally and importantly, transformation of large-insert BIBACs is particularly desirable for engineering genes from a species to an unrelated species, such as from cotton to Arabidopsis, because the native regulatory sequences and neighboring genes of the donor species are important to the transgene expression in the recipient species [22,24]. Therefore, a BIBAC library constructed from a cultivated cotton is of significance for cotton gene and QTL cloning [23], genome functional analysis and molecular breeding [22,24].

The genome origin and polyploidization of polyploid cottons, including two cultivated cottons (G. hirsutum and G. barbadense) and three wild species (G. tomentosum, G. mustelinum and G. darwinii), have been studied extensively [1-5]. A few extant diploid species containing an A or a D genome, including G. raimondii, have been proposed to be the most closest reluted of the polyploid cotton A- and D-subgenomes [4]. Nevertheless, debate widely exists about the genome origin and evolution of the polyploid cotton A- and D-subgenomes because such knowledge is of significance for enhanced cotton genetic improvement. The recent release of the G. raimondii draft genome sequence (http://www.ncbi.nlm.nih.gov/sra/SRA024364?report $=$ full) provides useful tools for advanced studies of the origin and evolution of the polyploid cotton A- and Dsubgenomes.

In this study, we constructed a large-insert BIBAC library from Upland cotton (G. hirsutum) cv. "Texas Marker-1" (TM-1) having a genome size of $2,425 \mathrm{Mb} / 1 \mathrm{C}$ [25], sequenced the ends of a large number of the BIBACs and preliminarily compared the genome of Upland cotton with 
the D genome of G. raimondii. Upland cotton represents approximately $95 \%$ of cotton growing in the world, and the genotype TM-1 has been widely used in cotton genomics and genetics research [14]. Therefore, the BIBAC library constructed from the genotype provides tools and resources for many aspects of cotton genomics, genetics and molecular biology research. From the BIBAC library, we isolated the BIBAC clones containing a number of genes of agronomic importance, such as those involved in fiber development (MYBB, MYBT2 and RDL1), fiber cellulose biosynthesis (CelA1, CelA3, CelA6, GhCesA2, GhIRX3, GhCesA3, and an unnamed Ces), seed fatty acid metabolism (FADO6) and host-nematode interaction (MIC3 and MIC1-15). Moreover, we also isolated the BIBAC clones potentially containing the genes conferring resistance to cotton bacterial blight pathogen, Xanthomonas campestris pv. malvacearum. Finally, we sequenced approximately 10,000 BIBAC ends (BESs) randomly selected from the library, with a sequence sample of $400 \mathrm{bp}$ in an average of every $250 \mathrm{~kb}$ along the Upland cotton genome, to provide some insights into the Upland cotton genome and its relationships with G. raimondii. Using the BESs, we estimated the composition of the Upland cotton cv. TM-1 genome, identified over 1,269 SSRs, of which 1,006 were new, and compared the genome sequence of Upland cotton with that of G. raimondii. These results significantly promote genomics and genetics research of Upland cotton, including integrative physical and genetic mapping, genome sequencing and functional analysis of the Upland cotton genome, and provide deeper insights into the mechanisms underlying the genome origin, variation and evolution of Upland cotton.

\section{Results}

\section{Construction of the BIBAC library}

We successfully constructed a high-quality BIBAC library for Upland cotton using the TM-1 megabase-sized nuclear DNA partially digested with BamH I (Invitrogen, Carlsbad, CA) in pCLD04541 [26,27], a widely-used, Agrobacteriummediated plant-transformation-competent BIBAC vector [22,28-35]. Cottons are extremely abundant in polyphenolics $[36,37]$ that may interact with DNA, thus interfering with DNA digestion and cloning $[36,38,39]$. Therefore, we prepared the DNA plugs from the cotyledons collected from the seedlings growing in a controllable growth chamber and treated in dark for $48 \mathrm{~h}$ (see the Methods). These measures minimized the contents of metabolites in the cells, especially polyphenolics and polysaccharides that may significantly influence the cloning of large DNA fragments. Furthermore, we washed the nuclei two more times than the original procedure for megabase-sized DNA preparation $[37,39,40]$. This measure further minimized the chloroplasts, mitochondria, polyphenolics and polysaccharides contained in the cytoplasm. Therefore, the resulting agarose plugs of the cotton nuclear DNA were transparent and pale in color, which is generally considered to be well suited for large-insert DNA library construction [38]. The third measure that we took to enhance the quality of the BIBAC library was the preparation of the DNA plugs at a proper concentration of DNA per plug. This is because the concentration of DNA in the plugs would significantly affect the methods and results of size selection on agarose gels, thus the insert sizes of the BIBAC clones resulted from the DNA [38]. Therefore, the use of the tissues containing reduced amount of polyphenolics and polysaccharides plus additional washes of nuclei before embedding into LMP agarose plugs is an effective and economical method to enhance the quality of megabase-sized DNA and ultimately the quality of the resultant BIBAC library. Preparation of the source DNA with an appropriate concentration in the agarose plugs further improved the average insert size of the resultant BIBAC library.

\section{Characterization of the BIBAC library}

The clones of the Upland cotton cv. TM-1 BIBAC library were individually arrayed into 200 384-well microtiter plates, containing a total of 76,800 clones. To determine the insert sizes of the clones and the portion of insertempty clones in the library, we selected a random sample of 122 clones from the library, isolated DNA from the clones, digested with Not I (Invitrogen) and analyzed on pulsed-field gels (Figure 1A). Four of the 122 clones (3.3\%) had no insert and the remaining 118 (96.7\%) all had inserts. The distribution of the clones according to their insert sizes is shown in Figure 1B. The clones had insert sizes ranging from 60 to $250 \mathrm{~kb}$, with an average insert size of $135 \mathrm{~kb}$. Of the clones, nearly $80 \%$ had insert sizes larger than $100 \mathrm{~kb}$. Furthermore, we estimated the percentage of the clones in the library derived from chloroplast DNA. A total of 691 positive clones were identified, accounting for approximately $0.9 \%$ of the library clones. Therefore, when the insert-empty clones and the chloroplast DNA-derived clones were excluded, approximately $95.8 \%(73,575)$ of the library clones contained inserts derived from cotton nuclear DNA. Since Upland cotton cv. TM-1 was estimated to have a genome size of $2,425 \mathrm{Mb} / 1 \mathrm{C}$ [25], the BIBAC library had a $4.1 \times$ coverage of the TM-1 haploid genome, with a $98.4 \%$ probability of obtaining at least one positive clone from the library using a single-copy probe $[41,42]$.

\section{Identification of BIBACs containing genes important in cotton}

To further characterize the library and test its utility for cotton genome research, we screened the library with 13 genes of agronomic importance (Additional file 1: Table S1), and four RGAs (resistance gene analogues) closely linked to the cotton bacterial blight resistance 


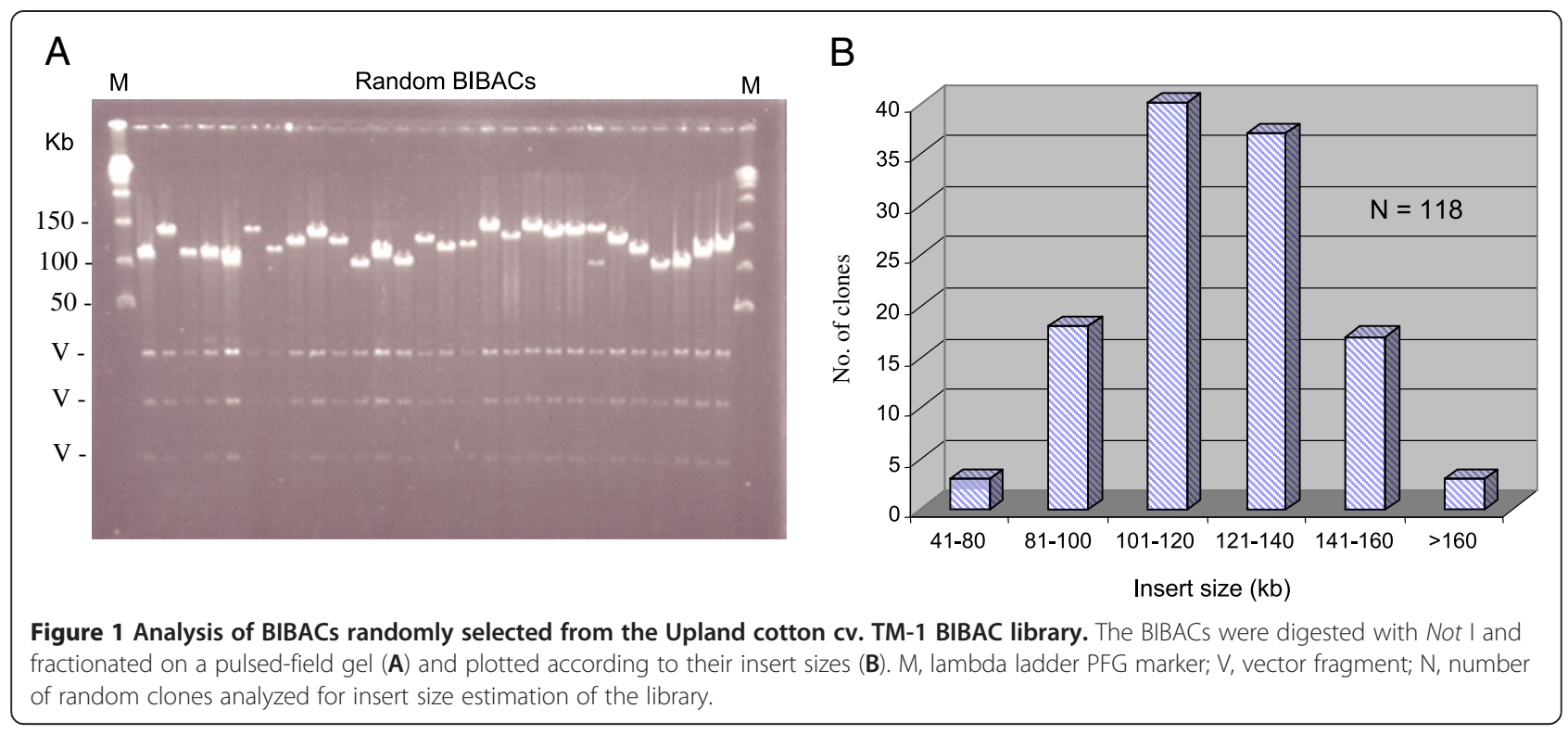

(Table 1). The library screening with the 13 genes resulted in a total of 60 positive clones, with each gene probe having 2-8 positive clones and an average number of 4.6 positive clones (Table 2), suggesting that the BIBAC library is suitable for map-based cloning and characterization of genes and QTLs important in cotton. The library screening with the four RGAs yielded 1,72 and 104 positive clones for 1 C08, 2D14 and 2D03/2B19, respectively (Table 1 ). Since the genome coverage of the library was $4.1 \times$, the sequences of 2D14 and 2D03/2B19 are possibly dispersed in as many as 17 and 25 loci, respectively, in the Upland cotton genome.

\section{BES analysis}

A total of 10,752 BIBAC ends randomly selected from the Upland cotton BIBAC library were sequenced. After base calling and trimming the E. coli and vector sequences, 9,711 (90.3\% successful rate) Q20 BESs with a minimum length of 50 bp were generated (dbGSS JY253441-JY262664). The total length of BESs was 3,842,009 bp, with each BES having a sequence length ranging from 50 to $842 \mathrm{bp}$, with an average length of 395 bp. Of these 9,711 BESs, 4,441 BIBAC clones $(84.3 \%)$ were successfully sequenced at both ends, generating mate-paired reads. Sequence analysis of the BESs indicated that the Upland cotton cv. TM-1 genome, as many other dicotyledonous plant genomes, is A/T-rich with $64.50 \% \mathrm{~A} / \mathrm{T}$ and $35.50 \% \mathrm{G} / \mathrm{C}$ (Table 3 ). From the 9,711 BESs, we identified a total of 2,912 or 3,022 exons, depending on the computer programs used (Table 3), and a total of 374,019 base pairs of repeated sequences. Of the transposable elements identified, the retroelement Gypsy/DIRS1 family alone accounted for $77.86 \%$ (4.22/ 5.42) (Table 4).

\section{Identification of microsatellites from the BESs}

To identify new SSR markers from the BESs, we analyzed the BESs for SSRs and compared the SSRs identified in this study with the existing cotton SSR database (www.cottonmarker.org). We identified a total of 1,269, 313 and 103 SSRs having motifs di-, tri-, tetra-, pentaand hexa-mers under the settings of $\geq 4, \geq 5$ and $\geq 6$ repeat units, respectively (Table 3). Analysis of the 1,269 SSRs showed that dimer SSRs absolutely predominate in the Upland cotton genome (Figure 2). Among the dimer SSR motifs, $(\mathrm{AT})_{\mathrm{n}}$ (where $n \geq 4$ ) was the most abundant in the genome, accounting for nearly $50 \%$ of all SSRs in the genome, and $(\mathrm{CT})_{\mathrm{n}},(\mathrm{AG})_{\mathrm{n}},(\mathrm{AC})_{\mathrm{n}}$ and $(\mathrm{GT})_{\mathrm{n}}$ were after $(\mathrm{AT})_{\mathrm{n}}$ in descending order, with each accounting for from $12.5 \%$ down to $5.5 \%$. The other types of SSR motifs together only accounted for approximately $15 \%$ of the SSRs in the genome. To determine how many of them were new, we downloaded the cotton SSR database that contains 17,343 SSRs from the Cotton Marker Database (www.cottonmarker.org). The 1,269 SSRs identified in this study were then searched against the cotton SSR database. As a result, the locus sequences of 263 of the 1,269 SSRs matched the cotton SSR database; therefore, 1,006 or $79.28 \%$ of the 1,269 SSRs were novel.

\section{Comparative sequence analysis of the polyploid Upland cotton genome with the diploid G. raimondii genome}

The origin of the D-subgenome of tetraploid cottons, including Upland cotton and Sea Island cotton, remains obscure, even though G. raimondii was previously proposed to be the closest diploid progenitor $[4,45]$. Because the whole genome sequence of G. raimondii has recently become available to the public, we compared the Upland cotton genome with the G. raimondii genome using the 
Table 1 Positive clones of the Upland cotton TM-1 BIBAC library identified by library screening with RGAs closely linked with the resistance to bacterial blight pathogen, Xanthomonas campestris pv. malvacearum [43,44]

\begin{tabular}{|c|c|c|c|c|c|}
\hline \multicolumn{6}{|c|}{ 2D03 (AY600401) and 2B19 (AY600394) (104 positive clones): } \\
\hline B004B14 & B011P19 & B013N15 & B017B02 & $\mathrm{B} 017 \mathrm{H} 07$ & B019104 \\
\hline B025M21 & B026F24 & B030122 & B041011 & B046C16 & $\mathrm{B} 046 \mathrm{H} 18$ \\
\hline B048P23 & B050K21 & B051D16 & B051D23 & B052F10 & B055119 \\
\hline B059G06 & B059N17 & B060P23 & B061B24 & B062117 & B063115 \\
\hline B063L01 & B065M06 & $\mathrm{B} 068 \mathrm{H} 24$ & B069J11 & B071B09 & B071D22 \\
\hline B072P14 & B075A15 & B076D08 & B080N11 & B085C16 & B085D09 \\
\hline B085G19 & B087G17 & B089E06 & B091L11 & B092C13 & B092P12 \\
\hline B093J03 & B097P19 & B104A01 & B105F15 & B105G23 & B108L23 \\
\hline B108P19 & B110J23 & B111C17 & B111K03 & B112G15 & B112K13 \\
\hline B117K22 & B118M16 & B119B09 & $\mathrm{B} 120 \mathrm{H} 01$ & B121B01 & B125002 \\
\hline B133A14 & B134N15 & B135C10 & B136C02 & B136019 & B138A12 \\
\hline B138A18 & B138D03 & B139E13 & B140B05 & B140J23 & B145L08 \\
\hline B147F15 & B148P17 & B151K12 & B155E18 & B160G13 & B161M18 \\
\hline B162E05 & $\mathrm{B} 162 \mathrm{H} 06$ & B163B09 & B165D12 & B169M17 & B170D18 \\
\hline B171A18 & B175N17 & B177F17 & B177M15 & B178C14 & B179B04 \\
\hline B179D06 & B179J12 & B179N18 & B184A18 & B186F07 & B188D16 \\
\hline B188E15 & B188G13 & B188|15 & B189B12 & B189J03 & B192018 \\
\hline B197K02 & B199N18 & & & & \\
\hline \multicolumn{6}{|c|}{ 2D14 (AY600383) (72 positive clones): } \\
\hline B006G23 & B007N18 & B008N22 & B017B02 & B017K11 & B022M07 \\
\hline B037|22 & B015L11 & B016M13 & B013N15 & B014N21 & B015O20 \\
\hline B011P19 & B025B03 & B032B23 & B028D04 & B030D15 & B026F24 \\
\hline B031122 & B042J09 & B047M01 & B045O16 & B048P23 & B050F06 \\
\hline B049K06 & B050K21 & B052L03 & B055P20 & B071D19 & B069E09 \\
\hline B069F11 & B069K14 & B070M07 & B066002 & B068P02 & B085C16 \\
\hline B085G19 & B057I10 & B061L07 & B059M03 & B061006 & B061O22 \\
\hline B075A15 & B080E20 & B077L10 & B075N13 & B073N21 & B096D03 \\
\hline B093J03 & B100J14 & B115E13 & B117K23 & B120M11 & B115P05 \\
\hline B133J15 & B107D12 & B111K03 & B142J06 & B148M10 & B166A18 \\
\hline B165D12 & $\mathrm{B} 163 \mathrm{H} 11$ & B161M18 & B184A18 & B181A23 & B183D05 \\
\hline B179002 & B154N23 & B171A18 & B175N14 & B176001 & B198J14 \\
\hline
\end{tabular}

1C08 (AY600376) (1 positive clone):

B125E12

9,711 BESs generated in this study as inquiries (Table 5). Surprisingly, only 57 of the $9,711(0.59 \%)$ Upland cotton BESs were aligned to the raimondii genome using the criteria of a continuous minimal match of $100 \mathrm{bp}$ and a sequence identity of $100 \%$. This number corresponded to only $0.41 \%$ of the total sequence length of the BESs attempted in the alignment. When the sequence identity was reduced to $90 \%$ or lower while the criterion of continuous minimal match of $100 \mathrm{bp}$ was maintained, the percentage of the Upland cotton BESs aligned to the raimondii genome reached and stabilized at approximately $70 \%$, corresponding to approximately $51 \%$ of the total inquired Upland cotton BES sequence length.

Moreover, we extracted the Upland cotton A- and Dsubgenome specific BESs from the 9,711 BESs using the A- or D-subgenome-derived BIBACs identified by Zhang et al. [46]. We were able to identify a total of 418 BESs derived from the A-subgenome BIBACs and 184 BESs derived from the D-subgenome BIBACs of Upland cotton (Table 5). When the A- and D-subgenome specific 
Table 2 The positive BIBAC clones of the genes identified by screening the Upland cotton cv. TM-1 BIBAC library using overgo probes designed from the gene sequences

\begin{tabular}{llc}
\hline Gene & Positive clones & No. of clones \\
\hline CelA1 & B130C07, B016N19 & 2 \\
CelA3 & B024E21, B072G17, B086A07, B083101, B073K01, B073L02, B096F08 & 7 \\
CelA6 & B098123, B116B12, B017105, B035E07, B115K16, B005M05, B086H21 \\
MIC3 & B099A19, B024H22, B014D05, B027E13, B026F22, B092E14 & 7 \\
MIC1-15 & B099A19, B024H22, B014D05, B027E13, B026F22, B092E14 \\
RDL1 & B175F03, B050B20, B187C03, B186M07, B016L07, B016L07, B166C05, B161G09 \\
FADO6 & B138P05, B080A19 & 6 \\
MYBB & B174C01, B192C23 & 6 \\
MYBT2 & B026F22, B007F03 & 8 \\
GhCe1A2 & B046G15,B048N17, B085P21, B162F12 \\
GhIRX3 & B070C20, B173F02 & 2 \\
GhCelA3 & B009G10, B065P05, B075L23, B097O20, B108L10, B146H15, B170C06, B178M15 \\
Unnamed Ces & B008D22, B145L18, B164E04, B165A23 & 2 \\
\hline
\end{tabular}

BESs were separately aligned to the G. raimondii genome sequence using the same criterion as above, the percentages of aligned BESs were not different from those obtained using the 9,711 BESs as inquiries. This result suggested that the genomic regions of Upland cotton from which the A- and D-subgenome specific BIBACs were derived have similar sequence identities to the other genomic regions of the A- and D-subgenomes with the G. raimondii genome. Since G. raimondii only has a $D$ genome $\left(D_{5}\right)$, it was expected that the percentage of the D-subgenome specific BESs aligned to the $G$. rainmodii genome would be much higher than that of the A-subgenome specific BESs. Surprisingly, no significant difference was obtained between the percentages of

\begin{tabular}{|c|c|}
\hline Total BESs sequenced & 10,752 \\
\hline Q20 (50-842 bp) & 9,711 \\
\hline Paired-ends & 4,441 \\
\hline GC level & $35.50 \%$ \\
\hline Total length & $3,842,009 \mathrm{bp}$ \\
\hline Average read of BESs & $395.63 \mathrm{bp}$ \\
\hline \multicolumn{2}{|l|}{ Number of exons: } \\
\hline GenScan & 3,022 \\
\hline GeneMark & 2,912 \\
\hline \multicolumn{2}{|c|}{ Microsatellite (di, tri, tetra, penta and hexa): } \\
\hline Four or more repeat units & 1,269 \\
\hline Five or more repeat units & 313 \\
\hline Six or more repeat units & 103 \\
\hline
\end{tabular}

the A- and D-subgenome specific BESs aligned to the G. raimondii genome.

\section{Discussion}

We have developed a large-insert BIBAC library for Upland cotton in a BIBAC vector (pCLD04541) that is competent for direct plant transformation via both Agrobacterium [29,30] and biolistic bombardment [22]. The library contains 76,800 clones, with an average insert size of $135 \mathrm{~kb}$. Therefore, the Upland cotton BIBAC library has a reasonably large average insert size. The 76,800 clones of the BIBAC library, when the insert-empty clones and the clones derived from chloroplast DNA are excluded, provide a $4.1 \times$ coverage of the Upland cotton haploid genome, with an approximate $99 \%$ probability of obtaining at least one positive clone from the library using a single-copy sequence probe. The results from the library screening with 13 gene-specific overgo probes designed from the unique sequences of genes, in which we obtained 2-8 positive clones for every probe, support the estimated genome coverage of the library, even though some of the probes might hybridize to two or more copies of the sequences because Upland cotton is an allotetraploid containing two homoeologous genomes, A- and Dsubgenomes. Importantly, differing from the existing cotton BAC libraries [11-15], this BIBAC library is the first one competent for direct plant transformation via both Agrobacterium [29,30] and biolistic bombardment [22]. The plant transformability of the BIBAC library streamlines the map-based cloning of cotton genes and QTLs (Introduction) and large-scale functional analysis of the cotton genome through high-throughput BIBAC transformation [22,24]. Therefore, the BIBAC library reported here will promote many aspects of cotton genome 
Table 4 Characteristics of repeat elements contained in the Upland cotton BIBAC end sequences (BESs)

\begin{tabular}{lccc}
\hline Elements & Number of elements & Length occupied (bp) & Percentage of the BES (\%) \\
\hline Retroelements & 776 & 208,081 & 5.42 \\
LINES: & 21 & 4,469 & 0.12 \\
$\quad$ L1/CIN4 & 21 & 4,469 & 0.12 \\
LTR elements: & 755 & 203,612 & 5.39 \\
$\quad$ Ty1/Copia & 166 & 40,878 & 1.06 \\
$\quad$ Gypsy/DIRS1 & 578 & 162,030 & 4.22 \\
DNA transposons & 5 & 302 & 0.01 \\
MuDR-IS905 & 3 & 152 & 0.00 \\
Total interspersed repeats & 208,383 & 5.42 & \\
Small RNA & 131 & 36,531 & 0.95 \\
Simple repeats & 201 & 10,777 & 0.28 \\
Low complexity & 2,289 & 118,328 & 3.08 \\
\hline
\end{tabular}

research, including map-based gene and QTL cloning, genome physical mapping, genome sequencing and functional analysis of the Upland cotton genome.

We have used the BIBAC library to isolate BIBACs containing or closely linked to the genes controlling fiber development, fiber cellulose biosynthesis, seed fatty acid metabolism, cotton-nematode interaction, and cotton bacterial blight resistance. This experiment has not only further verified the quality and demonstrated the utility of the BIBAC library for cotton genomics research, but also provided the tools essential for characterization of the genes at the genomic level and promoted the use of the genes in molecular breeding through BIBAC transformation. Using the BIBACs, we have developed a highthroughput system for functional analysis of the entire Upland cotton genome in the plant model species, Arabidopsis thaliana, with which the entire Upland genome could be transformed into Arabidopsis within approximately three years by one scientist (M.P. Z, Y.Z, D. M S, and H.-B. Z., unpublished).

Furthermore, the BESs generated in this study provide 9,711 STSs (sequence-tagged sites), with one STS in approximately every $250 \mathrm{~kb}$ along the Upland cotton genome. These STSs will facilitate integrative physical and genetic mapping, and sequencing of the Upland cotton genome using the next-generation sequencing technology $[47,48]$. Using the BIBAC library, we have constructed separate genome-wide physical maps for both the A- and D-subgenomes of Upland cotton cv. TM-1 and identified the minimal tiling path (MTP) clones (15,277 clones) spanning the physical map [46]. We have also initiated a project of sequencing the Upland cotton cv. TM-1 genome based on the BIBAC library using the nextgeneration sequencing technology (D.M. S., H.-B. Z.). We

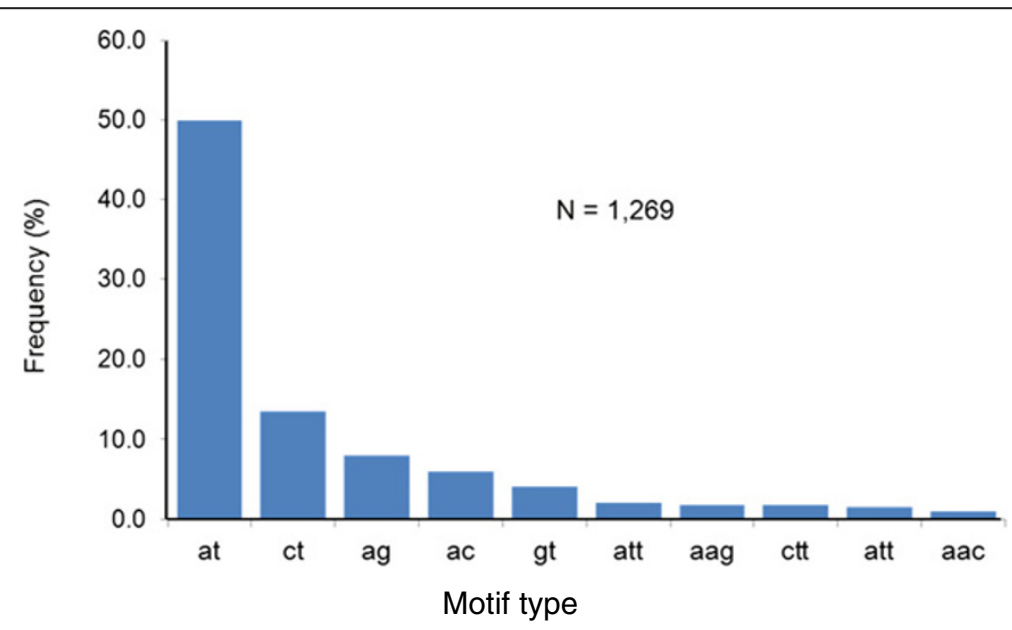

Figure 2 Frequency of the ten most abundant SSR motifs in the Upland cotton genome. The SSR motifs are di-, tri-, tetra-, penta- and hexa-mers, with four or more repeats. 
Table 5 Comparative sequence analysis of Upland cotton BESs with the D genome sequence of G. raimondii (DOE Joint Genome Institute, Cotton D V1.0, http://www.phytozome.net/cotton.php)

\begin{tabular}{|c|c|c|c|c|c|c|c|}
\hline \multirow[t]{2}{*}{ Inquired upland cotton BESs } & \multirow{2}{*}{$\begin{array}{l}\text { Criteria of the analysis } \\
\text { Continuous MinMatch (bp) }\end{array}$} & \multicolumn{6}{|c|}{ Sequence similarity } \\
\hline & & 100 & 100 & 100 & 100 & 100 & 100 \\
\hline All AD-subgenome BESs & Sequence identity (\%) & 100 & 95 & 90 & 80 & 70 & 60 \\
\hline \multirow{5}{*}{$\begin{array}{l}\text { (No. of } \mathrm{BESs}=9,711 \text {; total sequence } \\
\text { length }=3,842,009 \mathrm{bp} \text { ) }\end{array}$} & No. of BESs aligned & 57 & 4,587 & 6,588 & 7,118 & 7,257 & 7,277 \\
\hline & $\%$ of BESs aligned & 0.59 & 47.24 & 67.84 & 73.30 & 74.73 & 74.94 \\
\hline & $\begin{array}{l}\text { Total sequence length of BESs } \\
\text { aligned to the raimondii sequence } \\
\text { (bp) }\end{array}$ & 15,539 & $1,367,385$ & $1,960,413$ & $2,048,311$ & $1,996,820$ & $1,975,511$ \\
\hline & $\begin{array}{l}\% \text { of sequence length of BESs } \\
\text { aligned to the raimondii sequence }\end{array}$ & 0.41 & 35.59 & 51.10 & 53.31 & 51.95 & 51.42 \\
\hline & Continuous MinMatch (bp) & 100 & 100 & 100 & 100 & 100 & 100 \\
\hline A-subgenome-specific BESs & Sequence identity (\%) & 100 & 95 & 90 & 80 & 70 & 60 \\
\hline \multirow{3}{*}{$\begin{array}{l}\text { (No. of BESs }=418 \text {; total sequence } \\
\text { length }=169,533 \mathrm{bp} \text { ) }\end{array}$} & No. of BESs aligned & 7 & 205 & 294 & 310 & 317 & 317 \\
\hline & $\%$ of BESs aligned & 1.67 & 49.04 & 70.33 & 74.16 & 75.84 & 75.84 \\
\hline & Continuous MinMatch (bp) & 100 & 100 & 100 & 100 & 100 & 100 \\
\hline D-subgenome-specific BESs & Sequence identity (\%) & 100 & 95 & 90 & 80 & 70 & 60 \\
\hline \multirow{2}{*}{$\begin{array}{l}\text { (No. of BESs }=184 \text {; total sequence } \\
\text { length }=71,410 \mathrm{bp} \text { ) }\end{array}$} & No. of BESs aligned & 1 & 88 & 128 & 136 & 139 & 139 \\
\hline & $\%$ of BESs aligned & 0.54 & 47.83 & 69.57 & 73.91 & 75.54 & 75.54 \\
\hline
\end{tabular}

are developing the integrated physical and genetic maps of individual chromosomes of the Upland cotton genome, with 1-10 contigs per chromosome, from this BIBAC library and a BAC library, which will be necessary for assembling the chromosome-sized pseudomolecules of the Upland cotton genome sequence.

Analysis of the BESs has shown that the 9,711 BESs, with a length range from $50-842 \mathrm{bp}$ and an average length of approximately $400 \mathrm{bp}$, contain about 3,000 exons. If the exons of cotton genes have an average length of $200-500$ bp [49,50], the 3,000 exons are likely from approximately 3,000 genes, suggesting that approximately $30 \%$ of the 9,711 BESs are parts of genes. Among the transposable elements, the LTR retroelement family, Gypsy/DIRS1, is the most predominant in the Upland cotton genome, representing over $77 \%$ of the transposable elements in the genome. This result is consistent with that of Hawkins et al. [51] estimated for the genomes of its diploid putative progenitor species, $G$. raimondii and $G$. herbaceum, by random shotgun clone sequencing. The more than 1,000 new SSRs (79\% of the SSRs) identified from the BESs indicate the abundance of SSR loci in the Upland cotton genome and provide additional tools for cotton genome analysis, and gene and QTL mapping.

The comparative BES analysis has shown that the Upland cotton genome has significantly diverged from the G. raimondii genome. The unexpectedly high genome divergence between the two species could be attributed to the rapid evolution of the G. raimondii genome, the polyploidization and post-polyploidization evolution of Upland cotton, or both. However, the hypothesis of the rapid evolution of the G. raimondii genome does not appear to be compatible with the finding of this study that the A-subgenome of Upland cotton has the same level of similarity as its D-subgenome when compared to the G. raimondii genome, even though $G$. raimondii has only a D genome. Therefore, the rapid genome evolution after polyploidization must have occurred in Upland cotton, or both Upland cotton and G. raimondii. Moreover, it appears that an extensive element exchange has occurred between the A- and D-subgenomes of Upland cotton during the process of and/or after polyploidization, thus leading to a higher similarity between the two subgenomes at the genomic element sequence level. Therefore, when the A- and D-subgenome specific BESs were aligned to a third genome - the G. raimondii genome in this study, similar alignment results were obtained. Zhang et al. [5] studied the gene number variation of nucleotide-binding site (NBS)-encoding gene family and receptor-like kinase (RLK)-encoding gene family between the tetraploid cottons including Upland cotton and their diploid putative progenitor species including G. raimondii and G. herbaceum. They found that the tetraploid cottons have similar numbers of NBS and RLK genes as their diploid putative progenitor species, suggesting that a large number of genes in the families were lost during the process of and/or after the cotton polyploidizaiton. It has 
been found recently that most genome-constituent fundamental function elements of rice, including genes, DNA transposable elements, simple sequence repeats and low complexity repeats, have a very low content variation among different chromosomes even though they are nonhomologous, suggesting the existence of genomic element exchanges among chromosomes within a genome. These results provide indirect, but strong, support for the above hypothesis. (Liu Y-H, Zhang MP, Wu C, Huang JJ, Zhang $\mathrm{H}-\mathrm{B}$ : DNA is structured as a linear "Jigsaw Puzzle" in the genomes of Arabidopsis, rice and budding yeast, submitted for publication). However, further studies remain to determine the molecular mechanisms underlying the rapid genome evolution and genomic element exchanges between subgenomes in the polyploid cotton cells in the process of post-polyploidization.

\section{Conclusions}

We have constructed a high-quality large-insert planttransformation-competent BIBAC library for Upland cotton, isolated BIBACs containing several important cotton genes, sequenced nearly 10,000 BESs from BIBACs randomly selected from the cotton BIBAC library and identified over 1,000 new SSR markers from the BESs. These results provide resources and tools useful for advanced research of cotton genomics, genetics and breeding in numerous aspects such as Upland cotton genome integrative physical and genetic mapping, genome sequencing, gene and QTL cloning and characterization, and molecular breeding. Moreover, this study provides some insights into the genome composition and organization of the tetraploid cotton. The retroelement Gypsy/DIRS1 family and the SSRs with (AT) motifs are the most abundant in the Upland cotton genome. Finally, comparative sequence analysis has shown that the genome of the tetraploid Upland cotton has diverged much more than expected from that of its D-subgenome diploid putative progenitor species, $G$. raimondii. The divergence has likely resulted from the rapid evolution of tetraploid cotton and an apparent extensive genome-constituent element exchange or "balancing" between the A- and D-subgenomes of the tetraploid cotton during the process of postpolyploidization, even though further studies remain to test this hypothesis.

\section{Methods}

\section{Plant materials}

Upland cotton cv. TM-1 was used as the source DNA of the BIBAC library. The seeds of the cultivar were obtained from USDA/ARS, College Station, Texas, germinated and grown in a growth chamber at $25^{\circ} \mathrm{C}, 16 \mathrm{~h}$ light/8 h dark. Plant seedlings were treated in continuous dark for $48 \mathrm{~h}$ immediately before DNA isolation to minimize polyphenolics and polysaccharides. Cotyledons were harvested, frozen in liquid nitrogen and stored at $-80^{\circ} \mathrm{C}$ before use.

\section{Preparation of megabase-sized nuclear DNA}

Megabase-sized DNA was prepared according to Zhang et al. [37] with minor modifications [39,40,42,52]. Nuclei were washed for two additional times to minimize the contamination of polyphenolics, polysaccharides, chloroplasts and mitochondria contained in cytoplasm. The nuclei suspension of approximately $2 \times 10^{7}$ nuclei $/ \mathrm{ml}$ was prepared and used to make low-melting-point (LMP) agarose plugs. Therefore, each $100 \mu \mathrm{l}$-plug contained approximately $5 \mu \mathrm{g}$ nuclear DNA.

\section{Preparation of BIBAC vector}

The BIBAC vector pCLD04541 [27] was used for the construction of the BIBAC library. This vector has been widely used in the construction of BIBAC libraries for a number of species [22,28-35]. Vector DNA was isolated by the alkaline lysis method and purified by two rounds of the cesium chloride gradient centrifugation [38,53]. The vector DNA was completely digested with $\mathrm{BamH}$ I and prepared according to a procedure that we developed previously $[38,42]$.

\section{Library construction}

The nuclear DNA of Upland cotton was partially digested with BamH I and cloned in the BIBAC vector pCLD04541 to construct the BIBAC library [38]. To determine the optimal condition for partial digestion of megabase-sized DNA in LMP agarose plugs, particularly the amount of restriction enzyme $(\mathrm{BamH} \mathrm{I})$ per reaction and digestion incubation time, we first conducted a partial digestion test using a series of $B a m \mathrm{H}$ I concentrations per reaction. From the results of the partial digestion test, 2.4 units of $B a m \mathrm{H}$ I per reaction containing one-third of a $100-\mu \mathrm{l}$ megabase-sized DNA plug and 8 -min incubation at $37^{\circ} \mathrm{C}$ were selected for large-scale partial digestion for the library construction.

Ten 100- $\mu \mathrm{l}$ megabase-sized DNA plugs were used for partial digestion of the library construction using the condition predetermined by the partial digestion test (2.4 units of $\mathrm{BamH}$ I per reaction and 8 -min incubation at $37^{\circ} \mathrm{C}$ ). The partially digested megabase-sized DNA was subjected to one size selection on a $1 \%$ agarose gel by pulsed-field gel electrophoresis and DNA fragments ranging from 100$250 \mathrm{~kb}$ were selected [38]. The selected DNA fragments contained in the agarose gel were electroeluted, dialyzed against $0.5 \times$ TE (5 mM Tris-HCl, 0.5 mM EDTA, pH 8.0) and ligated into the dephosphorylated pCLD04541 vector. The ligation was performed at a molar ratio of 3 vectors: 1 insert, $1.5 \mathrm{ng}$ of insert DNA / $\mu \mathrm{l}$ of ligation reaction and 1.0 
unit T4 DNA ligase (Invitrogen) /50 $\mu \mathrm{l}$ of ligation reaction at $16^{\circ} \mathrm{C}$ for $8 \mathrm{~h}$.

The ligated DNA was transformed into Escherichia coli strain DH10B (Invitrogen) by electroporation using the Cell Porator ${ }^{\text {Th }}$ Device (Gibco BRL, Carlsbad, CA) consisting of Power Supply (cat. 1600), Chamber Safe (cat. 1600) and Voltage Booster (cat. 1612). The electroporation settings were as Zhang et al. [38], and 375 volts were used for electroporation. The transformed cells were selected for transformants and recombinant BIBACs on selective LB agar plates containing $15 \mu \mathrm{g}$ of tetracycline, $15 \mu \mathrm{g}$ of IPTG (isopropylthiogalactoside) and $60 \mu \mathrm{g}$ of X-Gal (5-bromo-4chloro-3-indolyl- $\beta$-D-galactoside) per $\mathrm{ml}$ of medium. Ligation selection and large-scale transformation for library construction were described as Zhang et al. [38]. The recombinant white clones were arrayed as individual BIBACs in 384-well microtiter plates, duplicated and kept at $-80^{\circ} \mathrm{C}$.

\section{Library characterization}

A random sample of the clones arrayed in the 384-well microtiter plates was analyzed [38] to estimate the insert sizes of the library clones and the percentage of clones having no inserts (insert-empty clones). BIBAC DNA was isolated, digested with Not I to release the insert of cotton DNA from the cloning vector pCLD04541 and subjected to pulsed-field gel electrophoresis. The insert size of each clone was estimated by the sum of the sizes of all insert band(s) in each gel lane using the lambda ladder PGE marker (New England BioLabs, Ipswich, MA) as the molecular weight marker.

The BIBAC library was printed in a $4 \times 4$ format using the GeneTAC Robotic Workstation (Genomic Solutions Inc., Ann Arbor, MI) onto $22.5 \times 22.5 \mathrm{~cm}$ Hybond N + membrane (Amersham-Pharmacia, Piscataway, NJ) laid on LB agar medium containing $15 \mu \mathrm{g}$ tetracycline $/ \mathrm{ml}$ medium. To facilitate reading the positive clones accurately and minimize the false positive clones, each of the library clones was printed in duplicate on the membrane. Therefore, each $22.5 \times 22.5$ membrane contained a total of $18,432(48 \times 384)$ double-printed clones and the entire library was printed on four $22.5 \times 22.5 \mathrm{~cm}$ and one $12 \times 8 \mathrm{~cm}$ membranes. The clones on the membranes were grown at $37^{\circ} \mathrm{C}$ overnight, the membranes were processed and the DNA of each clone was crosslinked on the membranes $[41,54]$. The membranes were hybridized with a probe prepared from a mixture of three chloroplast genes [41], $n d h \mathrm{~A}, r b c \mathrm{~L}$ and $p s b \mathrm{~A}$, to identify the clones of the library that were derived from chloroplast DNA. To ensure unambiguously reading the positive clones identified on the library membranes, we added $1-2 \mathrm{ng}[1-2 \%(\mathrm{w} / \mathrm{w})$ of the probe DNA] of the pCLD04541 vector DNA to the labeling reaction to create appropriate background when hybridized. The hybridization was done at $65^{\circ} \mathrm{C}$ overnight. After hybridization, the library membranes were washed in $1 \times$ SSC, $0.1 \%(\mathrm{w} / \mathrm{v})$ SDS [54] at $65^{\circ} \mathrm{C}$ twice, $10 \mathrm{~min}$ each time, followed by $0.5 \times \mathrm{SSC}, 0.1 \% \mathrm{SDS}$ at $65^{\circ} \mathrm{C}$ twice, 10 min each time.

\section{Identification of BIBACs containing genes important to cotton}

The membranes of the BIBAC library were screened to further verify its quality, demonstrate its utility and to identify the BIBACs containing cotton genes of interest. We first hybridized the library membranes using genespecific overgo probes to identify the BIBACs containing the genes involved in fiber development (MYBB, MYBT2 and RDL1), fiber cellulose biosynthesis (CelA1, CelA3, CelA6, GhCesA2, GhIRX3, GhCesA3 and an unnamed Ces), seed fatty acid metabolism (FADO6) and cottonnematode interaction (MIC3 and MIC1-15; Additional file 1: Table S1). The gene-specific overgos were designed from the unique sequences of the target genes, synthesized, pooled at equal amounts (100 ng per overgo pair) and used as probes to hybridize the library membranes [34]. The positive clones resulted from the primary hybridization were then re-arrayed into a new microtiter plate, double-printed onto a $12 \times 8 \mathrm{~cm}$ Hybond $\mathrm{N}+$ membrane in a $2 \times 2$ format and sorted according to individual gene overgos using a $4 \times 4$ pooling hybridization strategy $[55,56]$. To ensure unambiguously reading the positive clones identified on the library membranes, we added 1$2 \mathrm{ng}[1-2 \%(\mathrm{w} / \mathrm{w})$ of the gene overgos] of pCLD04541specific overgos (forward oligo 5'-TTAAGTTGGGTAAC GCCAGGGTTT-3 and reverse oligo 5'-CAACGTCGT GACTGGGAAAACCCT-3') to the gene-specific overgos. The gene and vector overgo mixture was labeled with $\left[{ }^{32} \mathrm{P}\right]$-dATP and $\left[{ }^{32} \mathrm{P}\right]-\mathrm{dCTP}$ to increase the hybridization signals, and the hybridization was carried out at $60^{\circ} \mathrm{C}$ for overnight. After hybridization, the library membranes were washed in $1 \times \mathrm{SSC}, 0.1 \%(\mathrm{w} / \mathrm{v}) \mathrm{SDS}$ at $60^{\circ} \mathrm{C}$ twice, $10 \mathrm{~min}$ each time, followed by $0.5 \times \mathrm{SSC}, 0.1 \% \mathrm{SDS}$ at $60^{\circ} \mathrm{C}$ twice, $10 \mathrm{~min}$ each time.

Moreover, we also hybridized the library membranes to identify the BIBACs containing the disease resistance analogues (RGAs) from three loci, 1C08 (GenBank No.: AY600376), 2D14 (AY600383), and 2D03 (AY600401)/ 2B19 (AY600394) [43]. The 1C08 RGA shares a significant similarity with the tomato root-knot nematode resistance gene, $\mathrm{Mi}-1.2[43,57]$ and is closely linked to the $B 2$ gene of the cotton bacterial blight (X. campestris pv. malvacearum) resistance [43,44]. The 2D14 and 2D03/ 2B19 RGAs flank the $\mathrm{Qb}_{6 \mathrm{a}}$ QTL of the bacterial blight resistance $[43,44]$. The inserts of the RGA clones were excised by enzymatic digestion and gel purification, labeled with $\left[{ }^{32} \mathrm{P}\right]$-dCTP using the random priming method and used as probes to hybridize the library membranes as the 
above library hybridization with the chloroplast gene probes.

\section{BIBAC end (BES) sequencing and analysis}

The BIBAC ends were sequenced according to $\mathrm{Li}$ et al. [58] with a few modifications using the Sanger method. Random BIBACs were inoculated into 96-deep well blocks containing $1.0 \mathrm{ml}$ of Terrific Broth medium per well and grown overnight in an incubator at $37^{\circ} \mathrm{C}$, $300 \mathrm{rpm}$. DNA was extracted using the alkaline lysis method [53] with modifications [59]. The DNA was dissolved in $15 \mu \mathrm{l} 0.5 \times \mathrm{TE}$. The sequencing reaction included $2 \mu \mathrm{l}$ BigDye Terminator v3.1 Cycle, $2 \mu \mathrm{l} 5 \times$ Sequence Buffer (Applied Biosystems, Carlsbad, CA), 300$500 \mathrm{ng}$ of template BIBAC DNA, $0.4 \mu \mathrm{l} 50 \mu \mathrm{mol} / \mathrm{L}$ primer (PCLD04541_T7: 5'-TAATACGACTCACTATAGGG-3' or PCLD04541_Rev primer: 5'-GAAAAGCTGGTACGTA $\left.-3^{\prime}\right)$ and distilled water added to $10 \mu \mathrm{l}$. The sequencing reaction was conducted by PCR at $95^{\circ} \mathrm{C}$ for $4 \mathrm{~min}$, followed by 99 cycles of $95^{\circ} \mathrm{C}$ for $15 \mathrm{~s}, 46^{\circ} \mathrm{C}$ for $10 \mathrm{~s}$ and $60^{\circ} \mathrm{C}$ for $4 \mathrm{~min}$. The sequencing reaction was purified by isopropanol precipitation, followed by two washes with $70 \%(\mathrm{v} / \mathrm{v})$ ethanol. Sequencing was carried out on ABI 3100 Genetic Analyzer (Applied Biosystems, Carlsbad, CA).

Software Phred $[60,61]$ was used for sequence base calling, with a quality score of Q20. The vector sequences were removed using Cross_match (http://www.phrap.org/ phredphrapconsed.html\#block_phrap). Sequence trimming was conducted by Sequencher v. 3.7 (Gene Codes Corp., Ann Arbor, MI). The repeat elements in the BESs were identified using RepeatMasker (A. Smit, G. Glusman and R. Hubley at http://repeatmasker.org) against the latest repeat library (as of 04/19/2011) of Arabidopsis thaliana that is the phylogenetically closest to cotton in the database, with the default parameters. We also predicted the genes/ exons contained in the BESs using Genscan [62] and GeneMark [63] against Arabidopsis model/smat using the default settings. The BESs were searched against the recently released G. raimondii genome sequence assembly (DOE Joint Genome Institute: Cotton D V1.0, http://www. phytozome.net/cotton.php) by a local Blat server [64] with a continuously minimal match of $100 \mathrm{bp}$ and a sequence identity of $100 \%, 95 \%, 90 \%, 80 \%, 70 \%$ and $60 \%$, respectively. Microsatellites were identified in non-redundant BESs using Msatfinder v2.0 that was specifically designed to identify and characterize microsatellites [65]. Only the microsatellites of 2-6 nucleotide motifs with at least 4, 5 and 6 repeat units were collected.

\section{Additional file}

Additional file 1: Table S1. Gene-specific overgos used for the Upland cotton CV. TM-1 BIBAC library screening.

\section{Abbreviations}

BIBAC: Binary bacterial artificial chromosome; BAC: Bacterial artificial chromosome; BES: BIBAC or BAC end sequence; SSR: Simple sequence repeat; RGA: Resistance gene analogue; EST: Expressed sequence tag; STS: Sequence-tagged site; QTL: Quantitative trait locus.

\section{Competing interests}

The authors declare that they have no competing interests.

\section{Authors' contributions}

$\mathrm{H}-\mathrm{BZ}$ conceived, designed and guided the study, and edited the manuscript. $M-K L, Y Z$ and MPZ performed experiments, including library construction, characterization, BES sequencing and analysis, and comparative sequence analysis. YZ wrote the manuscript while M-KL and MPZ edited the manuscript. HJK and BAT screened the library with some $\mathrm{Cel}$ genes and edited the manuscript. MG helped assemble and characterize the library. DMS guided the study and assisted in characterization of the library. All authors read and approved the final manuscript.

\section{Acknowledgements}

This study was supported by an internal fund of Zhang laboratory (20323286360) and the research grant of Texas AgriLife Research (124475-85360). We thank Chantel F. Scheuring for carefully reading and editing the manuscript.

\section{Author details}

'Department of Soil and Crop Sciences, 2474 TAMU, Texas A\&M University, College Station, TX 77843-2474, USA. ${ }^{2}$ College of Life Science, Jilin Agricultural University, Changchun, Jilin, China. ${ }^{3}$ USDA-ARS, Southern Regional Research Center, Cotton Fiber Bioscience, 1100 Robert E. Lee Blvd, New Orleans, LA 70124, USA.

Received: 14 July 2012 Accepted: 11 February 2013

Published: 28 March 2013

\section{References}

1. Wendel JF, Albert VA: Phylogenetics of the cotton genus (Gossypium L.): Character-state weighted parsimony analysis of chloroplast DNA restriction site data and its systematic and biogeographic implications. Syst Bot 1992, 17:115-143.

2. Seelanan T, Schnabel A, Wendel JF: Congruence and consensus in the cotton tribe. Syst Bot 1997, 22:259-290.

3. Small RL, Ryburn JA, Cronn RC, Seelanan T, Wendel JF: The tortoise and the hare: choosing between noncoding plastome and nuclear Adh sequences for phylogeny reconstruction in a recently diverged plant group. Amer J Bot 1998, 85:1301-1315.

4. Wendel JF, Cronn RC: Polyploidy and the evolutionary history of cotton. Adv Agron 2003, 87:139-186.

5. Zhang MP, Wu Y-H, Lee M-K, Liu Y-H, Rong Y, Santos FS, Wu C-C, Xie F, Nelson RL, Zhang H-B: Numbers of genes in the NBS and RLK families vary by more than four-fold within a plant species and are regulated by multiple factors. Nucleic Acids Res 2010, 38:6513-6525.

6. Pear JR, Kawagoe Y, Schreckengost WE, Delmer DP, Stalker DM: Higher plants contain homologs of the bacterial celA genes encoding the catalytic subunit of cellulose synthase. Proc Natl Acad Sci USA 1996, 93:12637-12642.

7. Delmer DP: Cellulose biosynthesis: exciting times for a difficult field of study. Annu Rev Plant Physiol Plant Mol Biol 1999, 50:245-276.

8. Richmond TA, Somerville CR: The cellulose synthase superfamily. Plant Physiol 2000, 124:495-498.

9. Yin $Y$, Huang J, $X u Y$ : The cellulose synthase superfamily in fully sequenced plants and algae. BMC Plant Biol 2009, 9:99.

10. Zhang H-B, Li Y, Wang B, Chee P: Recent advances in cotton genomics. Intern J Plant Genomics 2008, 2008:ID 742304.

11. Tomkins JP, Peterson DG, Yang TJ, Main D, Wilkins TA, Paterson AH, Wing RA: Development of genomic resources for cotton (Gossypium hirsutum L.): BAC library construction, preliminary STC analysis, and identification of clones associated with fiber development. Mol Breed 2001, 8:255-261.

12. Wang XF, Jun MA, Ma ZY, Zhang GY, Zheng YM: BAC library construction and characterization of Suyuan7235, a cotton germplasm with high fiber strength. J Cotton Sci 2006, 18:200-203. 
13. Yin J-M, Guo W-Z, Zhang T-Z: Construction and identification of bacterial artificial chromosome library for $0-613-2 R$ in upland cotton. $J$ Integrat Plant Biol 2006, 48:219-222.

14. Hu Y, Guo WZ, Zhang TZ: Construction of a bacterial artificial chromosome library of TM-1, a standard line for genetics and genomics in Upland cotton. J Integr Plant Biol 2009, 51:107-112.

15. Wang XF, Ma J, Wang WS, Zheng YM, Zhang GY, Liu CJ, Ma ZY: Construction and characterization of the first bacterial artificial chromosome library for the cotton species Gossypium barbadense L. Genome 2006, 49:1393-1398.

16. Lin L, Pierce GJ, Bowers JE, Estill JC, Compton RO, Rainville LK, Kim C, Lemke C, Rong J, Tang H, Wang X, Braidotti M, Chen AH, Chicola K, Collura K, Epps E, Golser W, Grover C, Ingles J, Karunakaran S, Kudrna D, Olive J, Tabassum N, Um E, Wissotski M, Yu Y, Zuccolo A, Rahman M, Peterson DG, Wing RA, Wendel JF, Paterson AH: A drafttphysical map of a D-genome cotton species (Gossypium raimondii). BMC Genomics 2010, 11:395

17. Hamilton CM, Frary A, Lewis C, Tanksley SD: Stable transfer of intact high molecular weight DNA into plant chromosomes. Proc Natl Acad Sci USA 1996, 93:9975-9979.

18. Frary A, Hamilton CM: Efficiency and stability of high molecular weight DNA transformation: an analysis in tomato. Transgenic Res 2001, 10:121-132.

19. Liu Y-G, Shirano Y, Fukaki H, Yanai Y, Tasaka M, Tabata S, Shibata D: Complementation of plant mutants with large genomic DNA fragments by a transformation-competent artificial chromosome vector accelerates positional cloning. Proc Natl Acad Sci USA 1999, 96:6535-6540.

20. Liu Y-G, Liu H, Chen L, Qiu W, Zhang Q, Wu H, Yang C, Su J, Wang Z, Tian $D$, Mei M: Development of new transformation-competent artificial chromosome vectors and rice genomic libraries for efficient gene cloning. Gene 2002, 282:247-255.

21. He R-F, Wang Y, Shi Z, Ren X, Zhu L, Weng Q, He G-C: Construction of a genomic library of wild rice and Agrobacterium-mediated transformation of large-insert DNA linked to BPH resistance locus. Gene 2003, 321:113-121.

22. Chang Y-L, Chuang H-W, Meksem K, Wu F-C, Chang C-Y, Zhang MP, Zhang $\mathrm{H}-\mathrm{B}$ : A plant-transformation-ready large-insert BIBAC library of Arabidopsis and bombardment transformation and expression of its large-insert BIBACs in tobacco. Genome 2011, 54:437-447.

23. Zhang H-B: Map-based cloning of genes and quantitative trait loci. In Principles and Practices of Plant Genomics, Vol.1: Genome Mapping. Edited by Kole C, Abbott AG. New Hampshire: Science Publishers; 2007:229-267.

24. Song R, Segal G, Messing J: Expression of the sorghum 10-member Kafirin gene cluster in maize endosperm. Nucleic Acids Res 2004, 32:e189.

25. Hendrix B, Stewart JM: Estimation of the nuclear DNA content of Gossypium species. Ann Bot 2005, 95:789-797.

26. Jones JDG, Shlumukov L, Carland F, English J, Scofield SR, Bishop GJ, Harrison K: Effective vectors for transformation, expression of heterologous genes, and assaying transposon excision in transgenic plants. Transgenic Res 1992, 1:285-297.

27. Tao Q, Zhang H-B: Cloning and stable maintenance of DNA fragments over $300 \mathrm{~kb}$ in Escherichia coli with conventional plasmid-based vectors. Nucleic Acids Res 1998, 26:4901-4909.

28. Meksem K, Ruben E, Zobrist K, Hyten D, Tao Q, Zhang H-B, Lightfoot DA: Two large-insert soybean genomic libraries constructed in a binary vector: Applications in chromosome walking and genome-wide physical mapping. Theor App/ Genet 2000, 101:747-755

29. Wu Y, Tulsieram L, Tao Q, Zhang H-B, Rothstein SJ: A binary vector-based large insert library for Brassica napus and identification of clones linked to a fertility restorer locus for Ogura cytoplasmic male sterility (CMS). Genome 2000, 43:102-109.

30. Men AE, Meksem K, Kassem MA, Lohar D, Stiller J, Lightfoot D, Gresshoff PM: A bacterial artificial chromosome library of Lotus japonicus constructed in an Agrobacterium tumefaciens- transformable vector. Mol Plant Microbe Interact 2001, 14:422-425.

31. Tao Q, Wang A, Zhang H-B: One large-insert plant-transformation -competent BIBAC library and three BAC libraries of japonica rice for genome research in rice and other grasses. Theor Appl Genet 2002, 105:1058-1066

32. Fang $X, G$, S, Xu Z, Chen F, Guo D, Zhang H-B, Wu N: Construction of a binary BAC library for an apomictic monosomic addition line of Beta corolliflora in sugar beet and identification of the clones derived from the alien chromosome. Theor Appl Genet 2004, 108:1420-1425.
33. Ortiz-Vázquez E, Kaemmer D, Zhang H-B, Muth J, Rodríguez-Mendiola M, Arias-Castro $C$, James A: Construction and characterization of a plant transformation-competent BIBAC library of the black Sigatoka-resistant banana Musa acuminata cv. Tuu Gia (AA). Theor Appl Genet 2005. 110:706-713.

34. Feng J, Vick BA, Lee $M-K$, Zhang $H-B$, Jan CC: Construction of BAC and BIBAC libraries from sunflower and identification of linkage groupspecific clones by overgo hybridization. Theor App/ Genet 2006, 113:23-32.

35. Zhang X, Scheuring CF, Zhang MP, Dong JJ, Zhang Y, Huang JJ, Lee M-K, Abbo S, Sherman A, Shtienberg D, Chen W, Muehlbauer F, Zhang H-B: A BAC/BIBAC-based physical map of chickpea, Cicer arietinum L. BMC Genomics 2010, 11:501.

36. Zhao X-P, Zhang H-B, Wing RA, Paterson AH: A simple method for cotton megabase DNA isolation. Plant Mol Biol Rep 1994, 12:126-131.

37. Zhang $H-B$, Zhao XP, Ding XD, Paterson AH, Wing RA: Preparation of megabase-sized DNA from plant nuclei. Plant J 1995, 7:175-184.

38. Zhang H-B, Scheuring CF, Zhang MP, Zhang Y, Wu C-C, Dong JJ, Li Y: Construction of BIBAC and BAC libraries from a variety of organisms for advanced genomics research. Nat Protocols 2012, 7:479-499.

39. Zhang MP, Zhang Y, Scheuring CF, Wu C-C, Dong JJ, Zhang H-B: Preparation of megabase-sized DNA from a variety of organisms using the nuclei method for advanced genomics research. Nat Protocols 2012, 7:467-478.

40. Zhang MP, Li Y, Zhang H-B: Isolation of megabase-sized DNA fragments from plants. In Handbook of Nucleic Acid Purification. Edited by Liu D. Florida: Taylor \& Francis Group, LLC; 2008:513-524.

41. Zhang H-B, Choi S, Woo SS, Li ZK, Wing RA: Construction and characterization of two rice bacterial artificial chromosome libraries from the parents of a permanent recombinant inbred mapping population. Mol Breed 1996, 2:11-24.

42. Wu C, Xu Z, Zhang H-B: DNA libraries. In Encyclopedia of Molecular Cell Biology and Molecular Medicine, Volume 3. 2nd edition. Edited by Meyers RA. Weinheim: Wiley-VCH; 2004:385-425.

43. He LM, Du CG, Covaleda L, Robinson AF, Yu JZ, Kohel RJ, Zhang H-B: Cloning, characterization, and evolution of the NBS-encoding resistance gene analogue family in polyploid cotton (Gossypium hirsutum L.). Mol Plant Microbe Interact 2004, 17:1234-1241.

44. Wright $\mathrm{R}$, Thaxton PM, El-Zik KM, Paterson AH: D-Subgenome Bias of $X \mathrm{~cm}$ Resistance genes in tetraploid Gossypium (cotton) suggests that polyploid formation has created novel avenues for evolution. Genetics 1998, 149:1987-1996.

45. Brubaker $\mathrm{CL}$, Paterson $\mathrm{AH}$, Wendel JF: Comparative genetic mapping of allotetraploid cotton and its diploid progenitors. Genome 1999, 42:184-203.

46. Zhang MP, Zhang Y, Huang JJ, Lee M-K, Zhang XJ, Stelly DM, Zhang H-B: Physical mapping of polyploid genomes: A BIBAC physical map of allotetraploid Upland cotton. PLOS One 2012, 7:e33644.

47. Steuernagel B, Taudien S, Gundlach H, Seidel M, Ariyadasa R, Schulte D, Petzold A, Felder M, Graner A, Scholz U, Mayer KFX, Platzer M, Stein N: De novo 454 sequencing of barcoded BAC pools for comprehensive gene survey and genome analysis in the complex genome of barley. BMC Genomics 2009, 10:247.

48. Sato K, Motoi Y, Yamaji N, Yoshida H: 454 sequencing of pooled BAC clones on chromosome 3H of barley. BMC Genomics 2011, 12:246.

49. The Arabidopsis Genome Initiative: Analysis of the genome sequence of the flowering plant Arabidopsis thaliana. Nature 2000, 408:796-815.

50. Feng Q, Zhang Y, Hao P, Wang S, Fu G, Huang Y, Li Y, Zhu J, Liu Y, Hu X, Jia $P$, Zhang $Y$, Zhao Q, Ying K, Yu S, Tang Y, Weng Q, Zhang L, Lu Y, Mu J, Lu Y, Zhang LS, Yu Z, Fan D, Liu X, Lu T, Li C, Wu Y, Sun T, Lei H, et al: Sequence and analysis of rice chromosome 4. Nature 2002, 420:316-320

51. Hawkins JS, Kim H, Nason JD, Wing RA, Wendel JF: Differential lineage-specific amplification of transposable elements is responsible for genome size variation in Gossypium. Genome Res 2006, 16:12521261.

52. Ren $C$, Xu ZY, Sun $S$, Lee M-K, Wu C, Scheuring C, Santos TS, Zhang H-B: Genomic DNA Libraries and Physical Mapping. In The Handbook of Plant Genome Mapping: Genetic and Physical Mapping. Edited by Meksem K, Kahl G. Weinheim: Wiley-VCH; 2005:173-213.

53. Sambrook J, Fritsch EE, Maniatis T: Molecular Cloning: Laboratory Manual. Cold Spring Harbor, New York: Cold Spring Harbor Laboratory Press; 1989.

54. Zhang H-B: Manual: Construction and Manipulation of Large-insert Bacterial Clone Libraries. College Station, Texas: Texas A\&M University; 2000 
55. Chang Y-L, Tao Q, Scheuring C, Meksem K, Zhang H-B: An integrated map of Arabidopsis thaliana for functional analysis of its genome sequence. Genetics 2001, 159:1231-1242.

56. Tao Q-Z, Chang Y-L, Wang J, Chen H, Scheuring C, Islam-Faridi MN, Wang B, Stelly DM, Zhang H-B: Bacterial artificial chromosome-based physical map of the rice genome constructed by restriction fingerprint analysis. Genetics 2001, 158:1711-1724.

57. Milligan SB, Bodeau J, Yaghoobi J, Kaloshianm I, Zabel P, Williamson VM: The root knot nematode resistance gene Mi from tomato is a member of the leucine zipper, nucleotide binding, leucine-rich repeat family of plant genes. Plant Cell 1998, 10:1307-1319.

58. Li Y, Uhm T, Ren C, Wu C, Santos TS, Lee M-K, Yan B, Santos F, Zhang A, Scheuring C, Sanchez A, Millena AC, Nguyen HT, Kou H, Liu D, Zhang H-B: A plant-transformation-competent BIBAC/BAC-based map of rice for functional analysis and genetic engineering of its genomic sequence. Genome 2007, 50:278-288.

59. Zhang $Y$, Zhang $X$, O'Hare TH, Payne WS, Dong JJ, Scheuring CF, Zhang MP, Huang JJ, Delany M, Zhang H-B, Dodgson JB: A comparative physical map reveals the pattern of chromosomal evolution between the turkey (Meleagris gallopavo) and chicken (Gallus gallus) genomes. BMC Genomics 2011, 12:447.

60. Ewing B, Hillier L, Wendl MC, Green P: Base-calling of automated sequencer traces using Phred. I. accuracy assessment. Genome Res 1998, 8:175-185.

61. Ewing B, Green P: Base calling of automated sequencer traces using Phred. II. error probabilities. Genome Res 1998, 8:186-194.

62. Burge C, Karlin S: Prediction of complete gene structures in human genomic DNA. J Mol Biol 1997, 268:78-94

63. Lomsadze A, Ter-Hovhannisyan V, Chernoff Y, Borodovsky M: Gene identification in novel eukaryotic genomes by self-training algorithm. Nucleic Acids Res 2005, 33:6494-6506.

64. Kent WJ: BLAT - the BLAST-like alignment tool. Genome Res 2002, 12:656-664

65. Thurston MI, Field D: Msatfinder: detection and characterisation of microsatellites. Mansfield Road, Oxford OX1 3SR: CEH Oxford; 2005. http:// www.genomics.ceh.ac.uk/msatfinder/.

doi:10.1186/1471-2164-14-208

Cite this article as: Lee et al:: Construction of a plant-transformation -competent BIBAC library and genome sequence analysis of polyploid Upland cotton (Gossypium hirsutum L.). BMC Genomics 2013 14:208.

\section{Submit your next manuscript to BioMed Central and take full advantage of:}

- Convenient online submission

- Thorough peer review

- No space constraints or color figure charges

- Immediate publication on acceptance

- Inclusion in PubMed, CAS, Scopus and Google Scholar

- Research which is freely available for redistribution 